\title{
Silencing of triggering receptor expressed on myeloid cells-2 enhances the inflammatory responses of alveolar macrophages to lipopolysaccharide
}

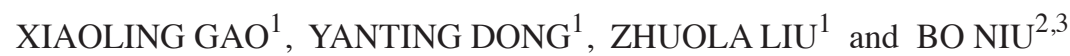 \\ ${ }^{1}$ Department of Respiratory, The Second Hospital of Shanxi Medical University; \\ ${ }^{2}$ Department of Biochemistry and Molecular Biology, Shanxi Medical University, Taiyuan 030001; \\ ${ }^{3}$ Biotechnology Laboratories, Capital Institute of Pediatrics, Beijing 100020, P.R. China
}

Received September 8, 2012; Accepted December 18, 2012

DOI: $10.3892 / \mathrm{mmr} .2013 .1268$

\begin{abstract}
Triggering receptor expressed on myeloid cells-2 (TREM-2) has been shown to attenuate inflammatory responses in various cell lines including bone marrow-derived macrophages, hepatic macrophages, osteoclasts and dendritic cells. However, its effects on alveolar macrophages remain unknown. Lentivirus-mediated RNA interference (RNAi) is a post-transcriptional gene silencing method, which is capable of degrading target genes specifically and efficiently. In this study, we silenced TREM-2 in murine alveolar macrophages by using lentivirus-mediated short hairpin RNA (shRNA) and evaluated the effects of TREM-2 silencing on expression of toll-like receptor-4 (TLR-4), tumor necrosis factor- $\alpha$ (TNF- $\alpha$ ) and interleukin-10 (IL-10) in response to lipopolysaccharide (LPS). Alveolar macrophages were transfected with shRNA targeting TREM-2 by use of lentivirus vector, non-sense shRNA as a negative control or empty lentivirus vector as a blank control. Silencing of TREM-2 was assessed by real-time fluorescence quantitative PCR and flow cytometry. Following LPS stimulation, the levels of TLR-4, TNF- $\alpha$ and IL-10 expressed in alveolar macrophages were measured by real-time PCR, flow cytometry or ELISA. TREM-2 expression on alveolar macrophages was downregulated significantly by lentivirus-mediated shRNA treatment at the transcriptional and translational levels. However, alveolar macrophages that received non-sense shRNA or empty lentivirus vectors showed no effects on TREM-2 expression. Silencing of TREM-2 enhanced expression of TLR-4, as well as TNF- $\alpha$ and IL-10, by alveolar macrophages following LPS stimulation. These results indicate a significant effect of TREM-2 on attenuating
\end{abstract}

Correspondence to: Dr Zhuola Liu, Department of Respiratory, The Second Hospital of Shanxi Medical University, No. 382 Wuyi Road, Taiyuan 030001, P.R. China

E-mail: zhuolaliu@yeah.net

Key words: lentivirus, triggering receptor expressed on myeloid cells-2, toll-like receptor- 4 , tumor necrosis factor- $\alpha$, interleukin-10 the LPS-induced inflammatory response of murine alveolar macrophages, which may be dependent on TLR-4.

\section{Introduction}

Alveolar macrophages, continuously exposed to various microbial pathogens and their products, are crucial for pulmonary defense. Lipopolysaccharide (LPS) is a major component of the cell wall in gram-negative bacteria and may lead to activation of inflammatory cells, including alveolar macrophages, through toll-like receptor 4 (TLR-4). TLR-4 is a member of the pattern recognition receptors, which are important in the clearance of microbial pathogens. Binding of TLR-4 results in the activation of both the myeloid differentiation factor 88 (MyD88)-dependent and MyD88-independent pathway, leading to the activation of nuclear factor $-\kappa \mathrm{B}(\mathrm{NF}-\kappa \mathrm{B})$, production of various inflammatory mediators and ultimately cellular injury (1). These mediators include tumor necrosis factor- $\alpha$ (TNF- $\alpha)$, interleukin (IL)-6, IL-2 and IL-10 $(2,3)$. The LPS-induced TLR-4 signaling pathway has been well studied, but its association with other receptors, such as triggering receptor expressed on myeloid cells (TREMs), and its molecular mechanism are not well defined. In this study, we investigated the correlation between TREM-2 and TLR-4 in alveolar macrophages in response to LPS, which may provide a new therapeutic strategy in the prevention and treatment of endotoxin-induced inflammatory response and cellular injury.

TREMs are also members of the pattern recognition receptors, playing important roles in regulating the immune response. TREM receptors include various members expressed mainly on immune cells of myeloid lineage (4). Of these members, TREM-1 and TREM- 2 are the most studied to date. TREM-1 is mainly expressed on neutrophils and monocytes, amplifying the inflammatory responses associated with sepsis $(5,6)$. Silencing of TREM-1 by siRNA significantly attenuates TLR-4 signaling by reducing the production of MyD88, CD14, IкB $\alpha$, IL-1 $\beta$, MCP-1 and IL-10 in macrophages treated with LPS (7). By contrast, TREM-2 is mainly expressed on macrophages, microglia, osteoclasts and dendritic cells and has been reported to negatively regulate immune responses (4). In TREM-2 $2^{-/}$mice, cytokine production by macrophages in 
response to LPS is upregulated; however, overexpression of TREM-2 in microglia leads to reduced production of TNF- $\alpha$ and inducible nitric oxide synthase (iNOS) $(8,9)$. This evidence indicates that TREM-1 enhances inflammatory responses and that TREM-2 plays an anti-inflammatory role in the regulation of the immune response. TREM-1 and TREM- 2 signal through a transmembrane adaptor molecule, DAP12, which contains an immunoreceptor tyrosine-based activation motif (ITAM). After initiation by the tyrosine phosphorylation of ITAM, DAP-12 signals to activate or suppress inflammatory responses, depending on which receptor it binds to $(4,10)$.

RNA interference (RNAi)-mediated gene silencing is now widely used for clinical and experimental purposes. RNAi was first recognized as a natural antiviral mechanism by which long double-stranded RNA (dsRNA) molecules produced during viral replication are cleaved by the endonuclease Dicer into short 21-23 nucleotide fragments known as small interfering RNAs (siRNAs). siRNAs are associated with a multiprotein nuclease complex known as the RNA-induced silencing complex (RISC), that selects and degrades mRNAs homologous to siRNAs, resulting in target gene silencing. siRNA can be delivered endogenously as a form of short-hairpin RNA (shRNA), which is generated by reverse complement of the siRNA sequence, and carried by plasmid or viral vectors into target cells and subsequently processed to siRNA. Compared with synthetic siRNA, the effects of shRNA are long-lasting, since it is continuously produced within the cells. Lentivirus vector is a newly developed shRNA vehicle, which is capable of transfecting various cell lines in both dividing and non-dividing phases with low immunogenicity, and integrates transgenes into the host genome, leading to long-term expression of the transgene and stable achievement of target gene silencing $(11,12)$.

In this study, we constructed a lentivirus-mediated shRNA system targeting TREM-2 in murine alveolar macrophages and investigated the effects of TREM-2 silencing on the expression of TLR-4, TNF- $\alpha$ and IL-10 in response to LPS treatment.

\section{Materials and methods}

Cell culture. Alveolar macrophages were harvested by bronchoalveolar lavage from healthy male C57BL/6 mice (8-12 weeks old, 20-25 g, provided by the Experimental Animal Research Institutes of Chinese Academy of Medical Sciences, China). Lungs were subjected to lavage 10 times with phosphate-buffered saline (PBS) $\left(37^{\circ} \mathrm{C}, 1 \mathrm{ml} /\right.$ time $)$. Subsequently, cells were obtained from the lavage fluid and $>95 \%$ of these cells were confirmed to be alveolar macrophages. More than 10 mice were used to obtain $6 \times 10^{6}$ alveolar macrophages for each group. These cells were divided into 6 wells per group. The isolated alveolar macrophages were cultured in RPMI-1640 medium (Gibco, Carlsbad, CA, USA) containing $10 \%$ fetal bovine serum, $100 \mathrm{U} / \mathrm{ml}$ penicillin and $100 \mu \mathrm{g} / \mathrm{ml}$ streptomycin (13). The study was approved by the ethics committee of Shanxi Medical University, Taiyuan, China.

Selection of TREM-2 shRNA. Taking into account the murine TREM-2 sequence from GenBank (accession no.: NM_031254), we designed two siRNA sequences and a scrambled siRNA using Ambion software (Ambion Life Technologies, Beijing, China): siRNA 573, 5'-GAU GCUGGA GAUCUCUGGG-3';
siRNA 737, 5'-GGAGGUACG UGAG AGAAUU-3'; scrambled siRNA, 5'-GGCACAAGC UGGAG UACAA-3'. A BLAST search was performed to prevent interference with non-specific genes. For shRNA construction, the sense and antisense sequences of siRNA were combined by a 9-base hairpin of the following sequence: 5'-TTCAAGAGA-3', and an HI promoter and RNA polymerase III termination sequence (TTTTT) were added. A double-stranded DNA oligonucleotide of these shRNA was annealed from two single-stranded ones and connected to the eukaryotic pGCsi plasmid (TransGen Biotech, China), which had been double digested by HindIII and BamHI (Takara, Japan). The recombinant plasmid was transfected into DH5 $\alpha$-competent E. coli cells (TransGen Biotech). A colony was selected for real-time polymerase chain reaction (RT-PCR) and the recombinant plasmids were extracted for sequence detection to ensure the accuracy of our design.

Moreover, alveolar macrophages were transfected with recombinant shRNA plasmids in 12-well plates for $48 \mathrm{~h}$ using TransFectin Lipid at the concentration indicated by the manufacturer's instructions (Bio-Rad, Hercules, CA, USA). The transfection efficiency was measured by flow cytometric analysis of green fluorescent protein (GFP), which was expressed by the pGCsi plasmid. mRNA of TREM-2 was extracted from transfected alveolar macrophages and detected by RT-PCR to select the optimal shRNA sequence.

Lentivirus-mediated shRNA expression system. shRNAs targeting TREM-2 were cloned into the lentiviral expression vector pLenti-EGFP-U6 (VGTC, Beijing, China), which was transfected into 293 T cells together with pLP1, pLP2 and $\mathrm{pLP} / \mathrm{VSVG}$ using a lentivirus packaging system and polybrene-gene companion II according to the manufacturer's instructions (VGTC). ELISA was used to test the virus titer. At $48 \mathrm{~h}$, packaged lentivirus particles were harvested, purified and extracted for sequence detection. As a negative control, a lentivirus vector containing scrambled shRNA was constructed and an empty vector without shRNA was used as a blank control.

Silencing of TREM-2 and LPS treatment of alveolar macrophages. The recombinant lentivirus vectors were delivered into alveolar macrophages in 24-well plates in the presence of polybrene (Sigma, St. Louis, MO, USA) at various concentrations. Each well contained $1 \times 10^{6}$ cells and these cells were cultured with LPS in RPMI-1640 medium in different conditions prior to or following recombinant lentivirus transfection. Alveolar macrophages were divided into 5 groups with triplicates per group: the TREM-2 shRNA+LPS group, the non-sense shRNA+LPS group, the empty vector+LPS group, the non-treatment group which underwent no interference and the LPS group which received LPS treatment alone. Cell viability was determined by MTT assay after transfection. RNA and protein samples were harvested at the indicated time points following treatment.

Real-time fluorescence quantitative PCR. We used real-time fluorescence quantitative PCR to evaluate mRNA expression of TREM-2 and TLR-4. Total RNA was extracted from alveolar macrophages using TRIzol reagent (Invitrogen, Carlsbad, CA, USA) according to the manufacturer's instructions. RNA (5 $\mu \mathrm{g})$ 


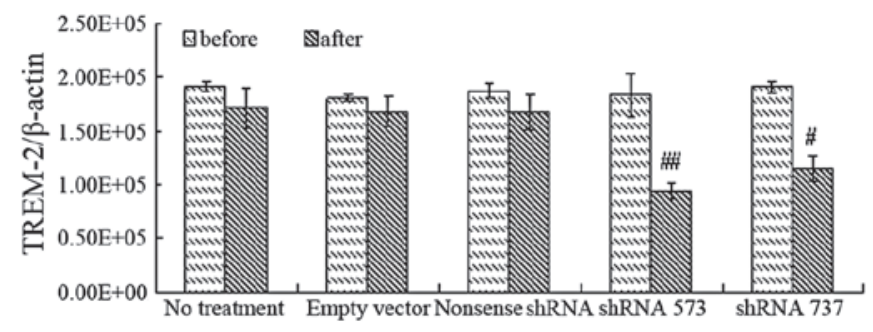

Figure 1. Silencing of TREM-2 in alveolar macrophages by recombinant eukaryotic plasmid vector-mediated shRNA. Alveolar macrophages were transfected with shRNA targeting TREM-2 or with non-sense shRNA or empty plasmid vector as controls. The effect of TREM-2 silencing was analyzed by RT-PCR. Data were presented as the means \pm SEM $(n=3),{ }^{~} \mathrm{P}<0.05$ compared with control, ${ }^{\# \#} \mathrm{P}<0.05$ compared with shRNA 737 group. TREM-2, triggering receptor expressed on myeloid cells-2; shRNA, short hairpin RNA.

was reverse transcribed to complementary DNA using a reverse transcription PCR Purification kit (Promega, Madison, WI, USA). The real-time PCR reaction was conducted in a total volume of $50 \mu \mathrm{l}$ with $10 \mu \mathrm{l}$ of cDNA templates. The housekeeping gene $\beta$-actin was used to normalize target gene expression. Primer sequences were as follows: 5'-TGGTCAGAGGGCTGGACTGT-3' (sense), 5'-TCCTGG CTGGACTTAAGCTGTAG-3' (antisense) for TREM-2 and 5'-TCAGAACTTCAGTGGCTGGATTTAT-3' (sense), 5'-TAGGGTTTCCTGTCAGTATCAAGTTTG-3' (antisense) for TLR-4. The primers for $\beta$-actin were 5'-AGCGGTTCC GATGCCCT-3' (sense) and 5'-AGAGGTCTTTACGGAT GTCAACG-3' (antisense). The reaction conditions were set as follows: an initial $2 \mathrm{~min}$ at $94^{\circ} \mathrm{C}$ followed by 40 cycles $(15 \mathrm{sec}$ at $94^{\circ} \mathrm{C}, 30 \mathrm{sec}$ at $60^{\circ} \mathrm{C}, 30 \mathrm{sec}$ at $72^{\circ} \mathrm{C}$ ) and a final $10 \mathrm{~min}$ at $72^{\circ} \mathrm{C}$. Quantification was performed by measuring the threshold cycle (CT value) and using the standard curve as a reference.

Flow cytometry. Flow cytometry was applied to detect protein levels of TREM-2 and TLR-4. Rat anti-mouse polyclonal antibody (R\&D Systems, Minneapolis, MN, USA) and goat anti-rat IgG conjugated to PE (Santa Cruz Biotechnology, Santa Cruz, CA, USA) were used as primary and secondary antibodies, respectively. The expression of TREM- 2 and TLR-4 by alveolar macrophages was measured by FACScan flow cytometry (BD Biosciences) and data were analyzed with CellQuest software.

ELISA. Levels of TNF- $\alpha$ and IL-10 in cell-free supernatant were determined by enzyme-linked immunosorbent assay (ELISA) (R\&D Co.) according to the manufacturer's instructions.

Statistical analysis. Data were analyzed using Statistical Package for Social Sciences version 14.0 for Windows (SPSS) software. Results were presented as the means \pm SEM. Analysis of variance (ANOVA) was applied to experiments with three or more groups. We used the SNK-q test to compare means between two groups. $\mathrm{P}<0.05$ was considered to indicate a statistically significant difference.

\section{Results}

Optimal sequence of TREM-2 shRNA. In this study, a eukaryotic pGCsi plasmid carrying TREM-2 shRNA was constructed

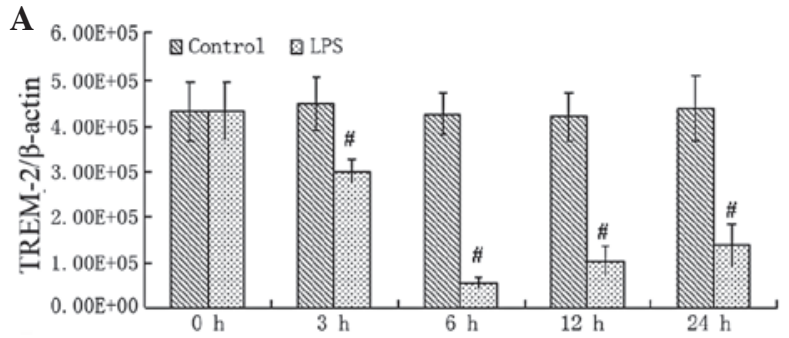

B
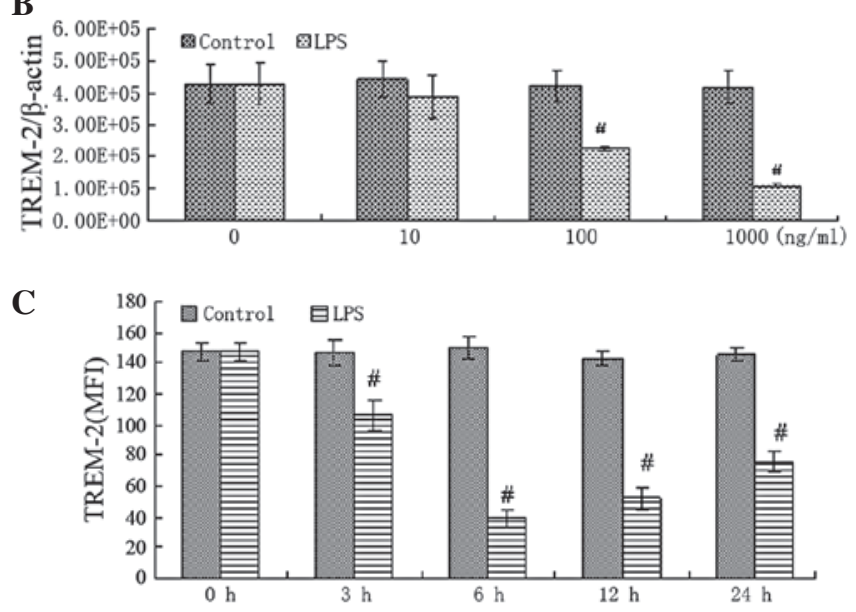

D

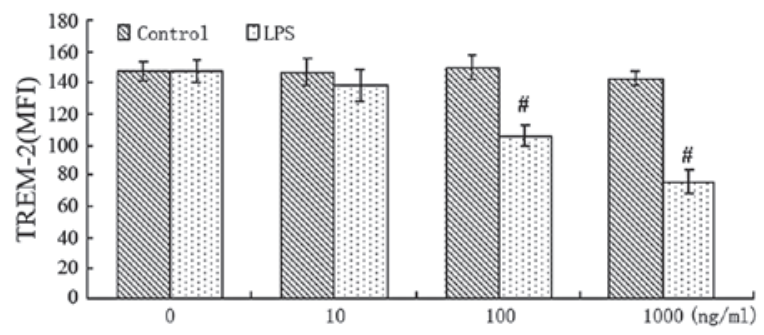

$\mathbf{E}$

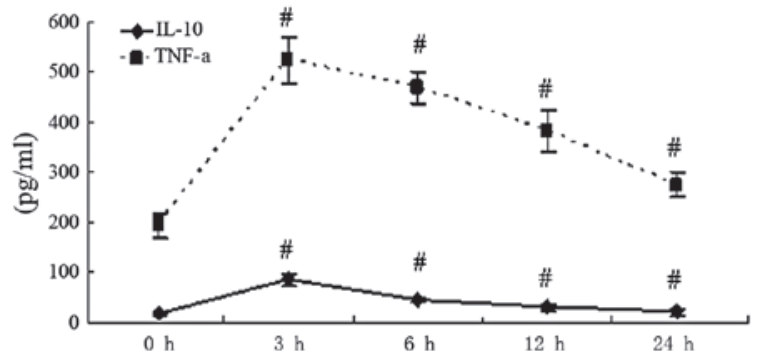

Figure 2. RT-PCR and flow cytometry analysis of TREM-2 expression in alveolar macrophages following LPS stimulation. (A) RT-PCR showed changes of TREM-2 mRNA in alveolar macrophages which were treated with $100 \mathrm{ng} / \mathrm{ml}$ LPS at various time points. (B) Alveolar macrophages were stimulated by LPS at different doses, and expression of TREM-2 mRNA was measured $3 \mathrm{~h}$ after LPS treatment by RT-PCR. (C and D) Flow cytometry analysis of TREM-2 protein and (E) ELISA analysis of TNF- $\alpha$ and IL-10 at indicated time points following treatment. Data were presented as the means \pm SEM $(n=3),{ }^{\#} \mathrm{P}<0.05$ compared with control. LPS, lipopolysaccharide; TREM-2, triggering receptor expressed on myeloid cells-2.

after selection from GenBank in order to determine the optimal target sequence for TREM-2 silencing. RT-PCR showed that the sequence of the recombinant plasmid was in accordance with our design. Alveolar macrophages were transfected by the recombinant plasmid with a transfection efficiency of $60.75 \pm 4.16 \%$, as measured by flow cytometry. Analysis of TREM-2 mRNA by RT-PCR suggested a higher inhibition effect of shRNA 573 than that of shRNA 737, while 
$\mathbf{A}$
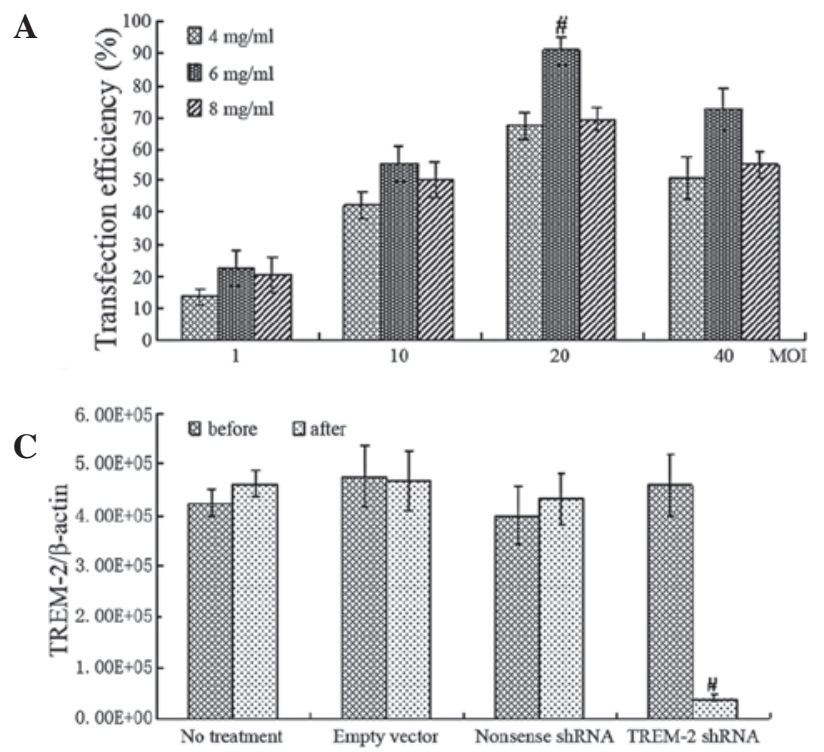
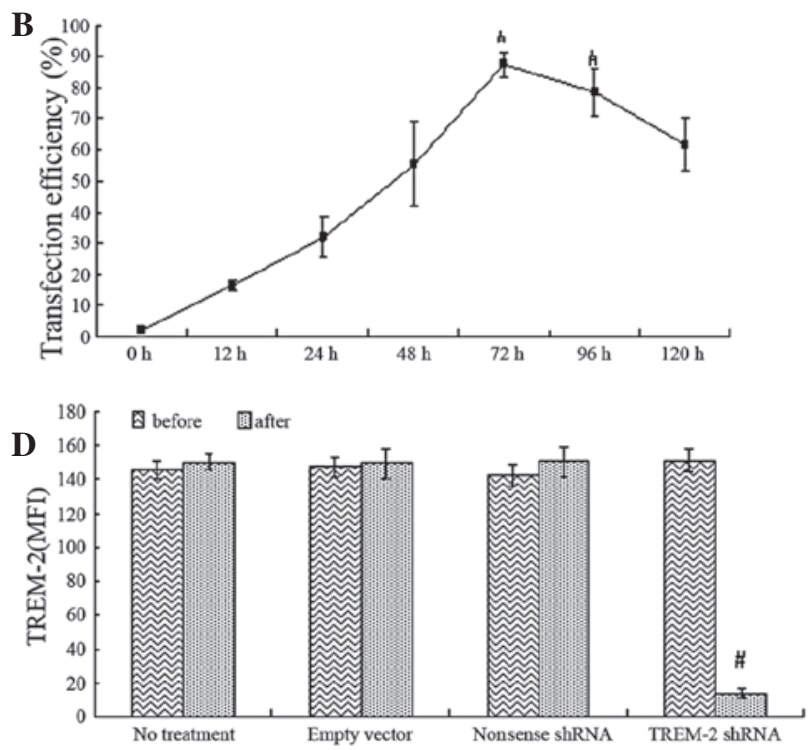

Figure 3. Suppression of TREM-2 gene and protein expression in alveolar macrophages by delivery of lentivirus-mediated TREM-2 shRNA. (A and B) Flow cytometry analysis of transfection efficiency with different multiplicity of infection (MOI, the number of lentivirus/well, the density of cells were $1 \times 10^{6} /$ well, $\mathrm{MOI}=1,10,20,40)$ and various doses of polybrene $(4,6$ and $8 \mathrm{mg} / \mathrm{ml})$ at different time points $(0-120 \mathrm{~h})$. (C and D) RT-PCR and flow cytometry showed levels of TREM-2 mRNA and protein in alveolar macrophages transfected with lentivirus-mediated shRNA or non-sense shRNA or empty lentivirus vector. Data were presented as the means \pm SEM $(n=6),{ }^{\#} \mathrm{P}<0.05$ compared with control. TREM-2, triggering receptor expressed on myeloid cells-2; shRNA, short hairpin RNA.

the scrambled shRNA and empty vector had no inhibitory effects on TREM-2 expression (Fig. 1). Therefore, shRNA 573 was selected as the target sequence for TREM-2 silencing.

LPS-induced inflammatory response. To evaluate how LPS affected TREM-2 expression in alveolar macrophages, mRNA and protein levels of TREM-2 were studied at different concentrations of LPS at various time points. RT-PCR showed that mRNA of TREM-2 was significantly downregulated $3 \mathrm{~h}$ after LPS treatment at a concentration of $100 \mathrm{ng} / \mathrm{ml}$. Increasing doses of LPS showed more significant reduction effects (Fig. 2A and B). Flow cytometry revealed similar changes in TREM-2 protein (Fig. $2 \mathrm{C}$ and D). Inflammatory mediators (TNF- $\alpha$ and IL-10) in the supernatants were highly upregulated $3 \mathrm{~h}$ after LPS treatment, as shown by ELISA (Fig. 2E). These results indicate that TREM-2 is downregulated, but TNF- $\alpha$ and IL-10 are upregulated significantly in response to LPS treatment.

TREM-2 expression. ELISA results demonstrated that the virus titer was $2.87 \times 10^{9} \mathrm{TU} / \mathrm{ml}$ in $293 \mathrm{~T}$ cells transfected with recombinant lentivirus-mediated shRNA. In the negative and blank control groups, virus titers were both $2.0 \times 10^{9} \mathrm{TU} / \mathrm{ml}$. Our study used flow cytometry to evaluate the transfection efficiency of lentivirus-mediated TREM-2 shRNA treatment in alveolar macrophages, and a time- and dose-dependent effect of transfection was observed. When the concentration of lentivirus was $2 \times 10^{7} \mathrm{TU} /$ well and polybrene was $6 \mu \mathrm{g} / \mathrm{ml}$ at $72 \mathrm{~h}$, the transfection efficiency reached the highest level (Fig. 3A and B) with cell viability of $89.2 \pm 3.2 \%$ as measured by the MTT method (Table I). RT-PCR showed a significant reduction of TREM-2 mRNA in alveolar macrophages treated with lentivirus-mediated shRNA, but not in the cells treated with non-sense shRNA or empty lentivirus vector (Fig. 3C).

Alveolar macrophages, cultured with LPS after delivery of lentivirus-mediated shRNA for $3 \mathrm{~h}$, showed a more significant
Table I. Cell viability (\%) $72 \mathrm{~h}$ after lentivirus-mediated TREM-2 shRNA treatment with different multiplicity of infection and doses of polybrene.

\begin{tabular}{cccc}
\hline & \multicolumn{3}{c}{ Concentration of polybrene } \\
\cline { 2 - 4 } MOI & $4 \mathrm{~g} / \mathrm{ml}$ & $6 \mu \mathrm{g} / \mathrm{ml}$ & $8 \mu \mathrm{g} / \mathrm{ml}$ \\
\hline 1 & $80.1 \pm 1.9$ & $75.5 \pm 2.4$ & $70.8 \pm 3.5$ \\
10 & $87.2 \pm 2.1$ & $88.5 \pm 2.1$ & $75.1 \pm 3.7$ \\
20 & $90.3 \pm 1.7$ & $89.2 \pm 3.2$ & $79.1 \pm 4.7$ \\
40 & $90.5 \pm 2.4$ & $91.2 \pm 4.1$ & $80.2 \pm 4.5$
\end{tabular}

The table shows that at $72 \mathrm{~h}$ after treatment with lentivirus-mediated TREM-2 shRNA, the cell viability of alveolar macrophages decreased as the concentration of polybrene increased. However, cell viability increased as the MOI increased. Cell viability was up to $89.2 \%$ with $\mathrm{MOI}=20$ and concentration of polybrene was $6 \mu \mathrm{g} / \mathrm{ml}$. Data were presented as the means $\pm \operatorname{SEM}(n=5)$.

reduction of TREM-2 gene expression than those in the LPS control group. By contrast, delivery of non-sense shRNA or empty vectors had no such effect (Fig. 4A). TREM-2 protein levels in transfected alveolar macrophages showed similar changes to the TREM-2 mRNA (Fig. 4B).

Silencing of TREM-2 upregulates TLR-4 expression. We investigated the effects of TREM-2 shRNA on levels of TLR-4 in response to LPS in murine alveolar macrophages. Levels of TLR-4 mRNA and protein were evaluated by RT-PCR and flow cytometry, respectively. We observed that TLR-4 gene expression was upregulated by LPS treatment. However, delivery of TREM- 2 shRNA increased levels of TLR- 4 mRNA more significantly at $3 \mathrm{~h}$ after LPS stimulation and delivery of 


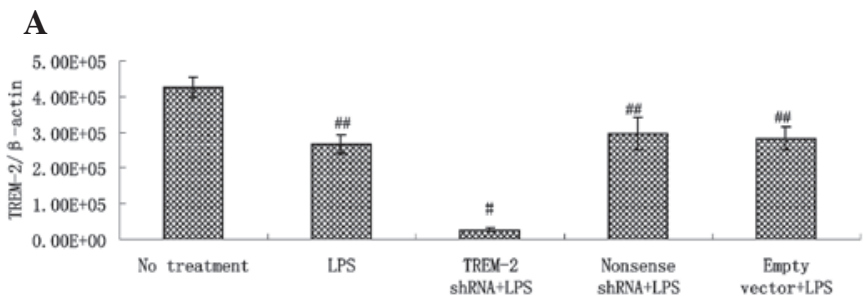

B

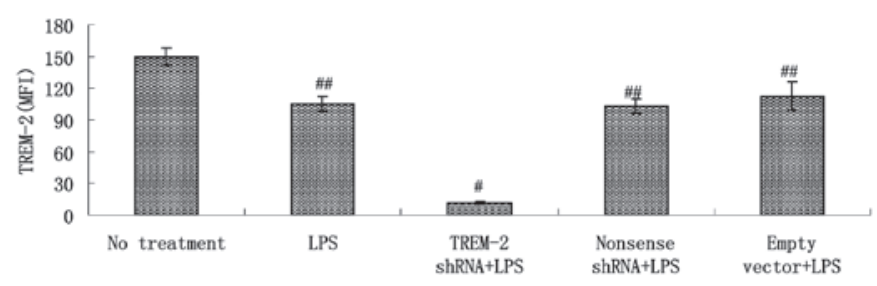

Figure 4. Inhibitory effects of lentivirus-mediated TREM-2 shRNA on TREM-2 expression in LPS-activated alveolar macrophages. (A) RT-PCR showed TREM-2 gene expression and (B) flow cytometry indicated changes of TREM-2 protein in alveolar macrophages. Data were presented as the means \pm SEM $(n=3),{ }^{\#} \mathrm{P}<0.05$ compared with LPS control group. ${ }^{\# \#} \mathrm{P}<0.05$ compared with the no treatment group. TREM-2, triggering receptor expressed on myeloid cells-2; shRNA, short hairpin RNA; LPS, lipopolysaccharide.

non-sense shRNA or empty lentivirus vector exhibited no such effect (Fig. 5A). Flow cytometry indicated similar changes in TLR-4 protein (Fig. 5B).

Effects of TREM-2 silencing on inflammatory mediators in response to LPS treatment. LPS stimulation increased levels of TNF- $\alpha$ and IL-10 in murine alveolar macrophages. Delivery of TREM-2 shRNA augmented expression of both TNF- $\alpha$ and IL-10 by $>1$-fold compared with the LPS control group, which indicates that silencing of TREM-2 significantly amplifies the production of TNF- $\alpha$ and IL-10 in response to LPS in murine alveolar macrophages (Fig. 5C).

\section{Discussion}

In this study, we have elucidated the contribution of TREM-2 to the inflammatory response induced by LPS and its correlation with TLR-4 in alveolar macrophages using lentivirus-mediated shRNA. The optimal sequence was selected for TREM-2 silencing and a recombinant lentivirus vector was constructed to deliver the selected shRNA into alveolar macrophages. Real-time fluorescence quantitative PCR and flow cytometry showed that TREM-2 was highly inhibited at the transcriptional and translational levels by use of lentivirus-mediated shRNA. Silencing of TREM-2 enhanced the expression of TLR-4 and the inflammatory mediators TNF- $\alpha$ and IL-10, which are recognized as downstream cytokines in the TLR-4 pathway. These results suggest that TREM-2 may suppress the inflammatory response through regulation of the TLR-4 pathway and this needs to be confirmed by further studies.

Lentivirus vector-mediated shRNA is a highly efficient RNAi technology with lower off-target effects and less endogenous interference with host genes than synthetic siRNA oligonucleotides $(11,12)$. For several years, lentivirus vector-mediated shRNA technology has been widely used
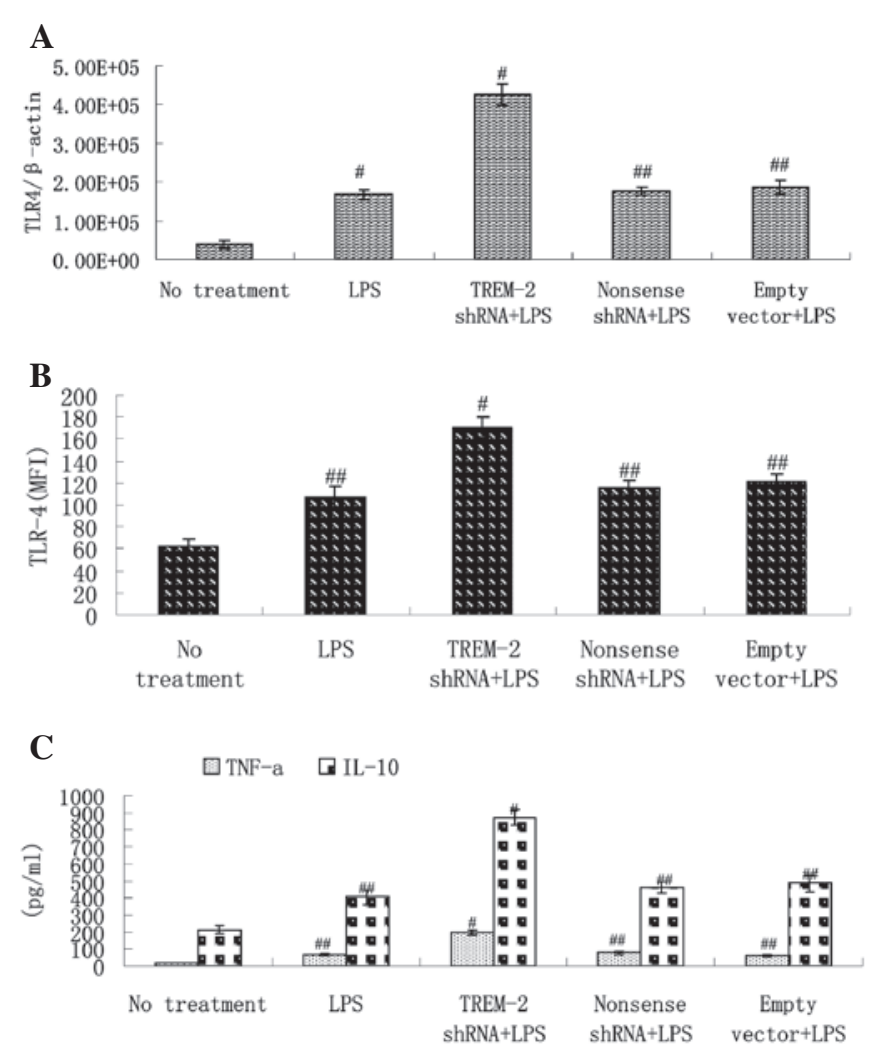

Figure 5. Silencing of TREM-2 enhances expression of TLR-4, TNF- $\alpha$ and IL-10 in LPS-stimulated alveolar macrophages. (A and B) TLR-4 mRNA and protein was detected by RT-PCR and flow cytometry and (C) inflammatory mediators TNF- $\alpha$ and IL-10 were measured by ELISA following TREM-2 silencing. Data were presented as the means \pm SEM $(n=3),{ }^{*} \mathrm{P}<0.05$ compared with LPS control group. ${ }^{\# \#} \mathrm{P}<0.05$ compared with no treatment control group. TREM-2, triggering receptor expressed on myeloid cells-2; LPS, lipopolysaccharide; TLR-4, toll-like receptor 4 ; TNF- $\alpha$, tumor necrosis factor- $\alpha$; IL-10, interleukin-10.

for its therapeutic potential in neurological disorders such as Alzheimer's disease (AD) $(12,14)$. Lentiviral vectors are capable of delivering long-term transgenes in various cell types in vivo and in vitro and suppress target genes in a specific manner, which provides broad applications in the study of genome functions and for clinical purposes $(11,12)$. However, there have been few studies applying this technology to TREM-2 in alveolar macrophages. In this study, we observed that lentivirus-mediated shRNA treatment significantly suppressed TREM-2 expression in alveolar macrophages.

The TREM family members, as innate immune receptors, play critical roles in modulating inflammatory responses, either by amplifying or dampening TLR-4-induced signaling (4). TREM-1 was established by initial findings as an amplifier of inflammatory responses and was also found to be associated with inflammatory bowel disease (IBD) $(4,15)$. On the contrary, TREM-2 is considered to be an important negative modulator of immune response. Previous studies have shown TREM-2 ligands on the surface of peritoneal and bone-marrow derived macrophages $(4,8)$. Others have reported that TREM-2 binds to anionic ligands on the surface of bacteria through Phexin-A and functions as a phagocytic receptor for bacteria $(4,16,17)$. Upon the binding of pathogens, TREM-2 is able to positively enhance phagocytosis and negatively suppress production of TNF- $\alpha$ and IL-6 in microglia in order to maintain the local 
immunosuppressive microenvironment in the central neural system (CNS) (9). Hamerman et al reported that TREM-2, associated with its counterpart DAP-12, inhibits TLR and FcR responses in bone-marrow-derived macrophages in vitro (18), and Turnbull et al showed that TREM-2 is expressed on newly differentiated and alternatively activated macrophages, functioning to restrain macrophage activation (8). In an animal model of LPS-induced acute lung injury (ALI), TREM-2 was highly decreased in lung tissues (19), which was accordant with our findings that TREM-2 levels in alveolar macrophages were significantly downregulated in response to LPS treatment. However, the exact mechanism by which TREM- 2 affects alveolar macrophages during LPS stimulation is not well defined.

TLR-4 has been established as a primary receptor for LPS, which initiates the production of pro-inflammatory mediators such as IL-1 $\beta$ and TNF- $\alpha$, leading to cellular injury and clinical manifestations of sepsis $(1,3)$. Previous studies suggest that TREM expression is associated with TLR-4 (20). Evidence has shown that activation of TREM-1 amplifies the TLR-4 signaling pathway by activating downstream mediators such as MyD88, CD14, IL-1 $\beta$, MCP-1, I $\mathrm{B} \alpha$ and IL-10 (7). In TLR-4 mutant $\mathrm{C} 3 \mathrm{H} / \mathrm{HeJ}$ mice, LPS treatment has little effect on both TREM-1 and TREM-2 in hepatic macrophages compared to TLR-4 normal cells in which TREM-1 expression was significantly increased, whereas TREM-2 was decreased (21). This evidence indicates that LPS-induced TREM alteration is dependent on TLR-4. As for TREM-2, production of TNF- $\alpha$ is inhibited in a TREM-2+DAP12 chimera in bone marrow-derived macrophages stimulated by TLR and FcR (including CpG DNA, zymosan and anti-Fc $\gamma \mathrm{RII} / \mathrm{III} \mathrm{mAb}$ ) (18). It is possible that the anti-inflammatory role of TREM-2 in LPS-induced immune responses occurs due to downregulation of TLR-4 and this is proved by the present study. Our study demonstrated that silencing of TREM-2 using recombinant lentivirus vector significantly upregulated levels of TLR-4 with LPS treatment. However, the mechanism remains to be further investigated.

After binding of LPS, TLR-4 initiates cascades of signaling, resulting in excessive production of inflammatory mediators including TNF- $\alpha$ and IL-10 (1,3). Turnbull et al have shown that peritoneal macrophages from TREM- $2^{-/}$mice produced increased TNF- $\alpha$ in response to LPS (8). Another study demonstrated that DAP-12-deficient macrophages treated with TREM-2+DAP12 chimeras inhibited the production of TNF- $\alpha$ with TLR and FcR stimulation (19). However, in regard to levels of IL-10 in response to LPS-induced inflammation, Turnbull et al observed no difference between wild-type and TREM-2-- macrophages (8).

In the present study, we demonstrated high levels of TNF- $\alpha$ and IL-10 but decreased expression of TREM-2 in alveolar macrophages after LPS stimulation. By silencing TREM-2, production of TNF- $\alpha$ and IL-10 were increased $>1$-fold. These results indicate that TREM-2 is reduced in the early stage of LPS-induced inflammation in order to increase the production of inflammatory mediators and activate the immune response. Silencing of TREM-2 leads to excessive production of inflammatory mediators, which aggravates the inflammatory response induced by LPS. This implies that TREM-2 is one of the major signaling pathways that negatively regulates the production of inflammatory mediators, which is consistent with the findings in other studies $(4,8,18)$.
In conclusion, this study has shown that silencing of TREM-2 activated the expression of TLR-4 and its downstream cytokines TNF- $\alpha$ and IL-10 in response to LPS in alveolar macrophages. Moreover, the anti-inflammatory effect of TREM-2 may be dependent on the TLR-4 pathway, and this requires further study.

\section{References}

1. Dauphinee SM and Karsan A: Lipopolysaccharide signaling in endothelial cells. Lab Invest 86: 9-22, 2006.

2. Chen LC, Gordon RE, Laskin JD and Laskin DL: Role of TLR-4 in liver macrophage and endothelial cell responsiveness during acute endotoxemia. Exp Mol Pathol 83: 311-326, 2007.

3. Adib-Conquy M, Moine P, Asehnoune K, et al: Toll-like receptormediated tumor necrosis factor and interleukin-10 production differ during systemic inflammation. Am J Respir Crit Care Med 168: 158-164, 2003.

4. Ford JW and McVicar DW: TREM and TREM-like receptors in inflammation and disease. Curr Opin Immunol 21: 38-46, 2009.

5. Bouchon A, Facchetti F, Weigand MA and Colonna M: TREM-1 amplifies inflammation and is a crucial mediator of septic shock. Nature 410: 1103-1107, 2001.

6. Zanzinger K, Schellack C, Nausch N and Cerwenka A: Regulation of triggering receptor expressed on myeloid cells 1 expression on mouse inflammatory monocytes. J Immunol 128: 185-195, 2009.

7. Ornatowska M, Azim AC, Wang X, et al: Functional genomics of silencing TREM-1 on TLR4 signaling in macrophages. Am J Physiol Lung Cell Mol Physiol 293: 1377-1384, 2007.

8. Turnbull IR, Gilfillan S, Cella M, et al: Cutting edge: TREM-2 attenuates macrophage activation. J Immunol 177: 3520-3524, 2006.

9. Takahashi K, Rochford CD and Neumann H: Clearance of apoptotic neurons without inflammation by microglial triggering receptor expressed on myeloid cells-2. J Exp Med 201: 647-657, 2005.

10. Lanier LL: DAP10- and DAP12-associated receptors in innate immunity. Immunol Rev 227: 150-160, 2009.

11. Manjunath N, Wu H, Subramanya S and Shankar P: Lentiviral delivery of short hairpin RNAs. Adv Drug Deliv Rev 61: 732-745, 2009.

12. Singer O and Verma IM: Applications of lentiviral vectors for shRNA delivery and transgenesis. Curr Gene Ther 8: 483-488, 2008.

13. Egan PJ, Andrews AE, Barcham GJ, Brandon MR and Nash AD: The extraction, purification and activity detection of murine alveolar macrophages. The public health of China 19: 1087-1088, 2003.

14. Singer O, Marr RA, Rockenstein E, et al: Targeting BACE1 with siRNAs ameliorates Alzheimer disease neuropathology in a transgenic model. Nat Neurosci 8: 1343-1349, 2005.

15. Schenk M, Bouchon A, Seibold F and Mueller C: TREM-1expressing intestinal macrophages crucially amplify chronic inflammation in experimental colitis and inflammatory bowel diseases. J Clin Invest 117: 3097-3106, 2007.

16. Daws MR, Sullam PM, Niemi EC, Chen TT, Tchao NK and Seaman WE: Pattern recognition by TREM-2: binding of anionic ligands. J Immunol 171: 594-599, 2003.

17. N'Diaye EN, Branda CS, Branda SS, et al: TREM-2 (triggering receptor expressed on myeloid cells 2 ) is a phagocytic receptor for bacteria. J Cell Biol 184: 215-223, 2008.

18. Hamerman JA, Jarjoura JR, Humphrey MB, Nakamura MC, Seaman WE and Lanier LL: Cutting edge: inhibition of TLR and FcR responses in macrophages by triggering receptor expressed on myeloid cells (TREM)-2 and DAP12. J Immunol 177: 2051-2055, 2006.

19. Sun GY, Guan CX, Zhou Y, et al: Vasoactive intestinal peptide re-balances TREM-1/TREM-2 ratio in acute lung injury. Regul Pept 167: 56-64, 2011.

20. Klesney-Tait J and Colonna M: Uncovering the TREM-1-TLR connection. Am J Physiol Lung Cell Mol Physiol 293: 1374-1376, 2007.

21. Chen LC, Laskin JD, Gordon MK and Laskin DL: Regulation of TREM expression in hepatic macrophages and endothelial cells during acute endotoxemia. Exp Mol Pathol 84: 144-145, 2008. 